\section{MS11-O3 Analysis of flexible multidomain glycoproteins with SAXS, analytical ultracentrifugation, and torsion-angle molecular dynamics}

Jordi Bella ${ }^{1}$, Thomas A. Jowitt ${ }^{1}$, Clair Baldock ${ }^{1}$, David Garrod ${ }^{1}$, Lydia Tabernero $^{1}$

1. Faculty of Life Sciences, University of Manchester, Manchester M13 9PT

email: jordi.bella@manchester.ac.uk

The extracellular regions of many cell-surface receptors and extracellular proteins are built from tandems of domains (immunoglobulin-like, cadherin, EGF-like, fibronectin type-III, etc) that are connected by inter-domain linkers of variable flexibility. This conformational flexibility, often combined with glycosylation, makes structural analysis of these multi-domain proteins particularly challenging. When high-resolution methods fail, biological small-angle X-ray scattering (SAXS) can provide at least some structural information about these molecules, mainly in terms of overall shape and dimensions. Drawbacks are the low resolution and limited amount of data, which can easily lead to overfitting or non-meaningful solutions that fit the data equally well. SAXS analysis of multi-domain proteins (or glycoproteins) often involves exploring their conformational space by generating a library of thousands of conformers, built from atomic models typically based on high-resolution crystal structures of homologous proteins. These confomers are then tested in turn against the SAXS data, and ensembles of best-fitting conformers are generated to describe the flexible states of these multi-domain proteins in solution (Tria et al, 2015, IUCrJ, 2: 207-17). Torsion-angle molecular dynamics, as implemented in the popular crystallographic package CNS (Brunger et al, 1998, Acta Cryst. D54: 905-21) offers an attractive route for exploring the conformational space of multi-domain proteins with much smaller libraries of conformers, thus requiring a smaller computational effort. In addition, it provides a more precise control of the flexibility between domains, allows for simultaneous sampling of the protein and sugar components of glycoproteins, and overcomes topological limitations for the definition of the flexible and rigid elements of the multidomain protein or glycoprotein being explored. Hydrodynamic data obtained from analytical ultracentrifugation can be combined with this conformational sampling to filter out conformers with unrealistic shapes and dimensions prior to their fitting to the SAXS data. This powerful combination increases the confidence in the SAXS analysis and produces more realistic models. Applications of this methodology will be illustrated with successful examples from our laboratory (Tariq et al, 2015, PNAS in press).

Keywords: SAXS, molecular dynamics, analytical ultracentrifugation, hydrodynamic modeling
MS11-04 Solving complex zeolite structures by combining electron crystallography, solid state NMR and powder X-ray diffraction

Xiaodong Zou ${ }^{1}$, Tom Willhammar ${ }^{1}$, Allen W. Burton ${ }^{2}$, Yifeng Yun ${ }^{1}$, Junliang Sun ${ }^{1}$, Mobae Afeworki ${ }^{2}$, Karl G. Strohmaier ${ }^{2}$, Hilda Vroman ${ }^{2}$

1. Berzelii Centre EXSELENT on Porous Materials and Inorganic and Structural Chemistry, Department of Materials and Environmental Chemistry, Stockholm University, SE-106 91 Stockholm, Sweden

2. Corporate Strategic Research, ExxonMobil Research \& Engineering Co. Inc, 1545 Route 22 East, Annandale, New Jersey 08801, USA

email: xzou@mmk.su.se

Zeolites are crystalline, microporous aluminosilicates that are used as catalysts or adsorbents in a wide array of industrial processes. Stable, multidimensional and extra-large pore zeolites are desirable by industry for catalysis and separation of bulky molecules. Here we report EMM-23, the first stable, three-dimensional extra-large pore aluminosilicate zeolite[1]. The structure of EMM-23 was determined from sub-micron sized crystals by combining electron crystallography, solid state NMR and powder $\mathrm{X}$-ray diffraction.

The polycrystalline nature, the large unit cell and especially the presence of $\mathrm{Q}^{2}$ and $\mathrm{Q}^{3} \mathrm{Si}$ species make the structure determination of EMM-23 challenging. Electron crystallography has unique advantages for structure solution of nano- or micron-sized crystals[2]. We applied the recently developed the rotation electron diffraction (RED) method[3] and collected 252 ED fames covering a tilt range of $63.74^{\circ}$ (Fig. 1). The crystallographic phase information from high resolution transmission electron microscopy (HRTEM) images has been used to facilitate the space group determination. The structure was solved from the RED data using direct methods, and refined by powder X-ray diffraction. Solid state NMR indicated that the zeolite possesses a high density of $\mathrm{Q}^{2}$ and $\mathrm{Q}^{3}$ silicon species, which was not observed before in zeolites. The solid state NMR data provided important information about the possible disorders in the structure, which were used for the structure refinement. The framework contains highly unusual tri-lobe shaped pores that are bound by $21-24 \mathrm{Si} / \mathrm{Al}$ atoms. These extra-large pores are intersected perpendicularly by a $2 \mathrm{D}$ 10-ring channel system. EMM-23 is stable after calcination at $540^{\circ} \mathrm{C}$.

\section{References}

[1] T. Willhammar, A.W. Burton, Y. Yun, J. Sun, M. Afeworki, K.G. Strohmaier, H. Vroman, X. Zou. J. Am. Chem. Soc. 2014, 136, 13570.

[2] X. Zou, S. Hovmöller, P. Oleynikov, Electron Crystallography, IUCr texts on crystallography, Oxford University Press, 2011.

[3] W. Wan, J. Sun, J Su, S. Hovmöller, X. Zou. J. Appl. Cryst. 2013, 46, 1863. 


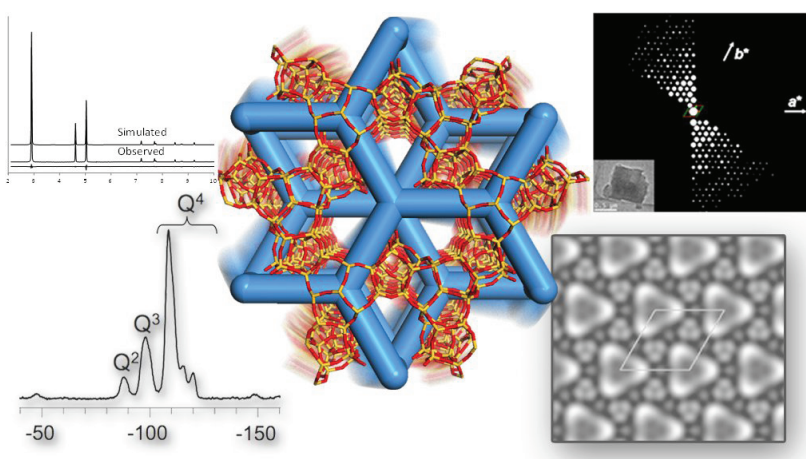

Figure 1. The structure of zeolite EMM-23 with the 3D channel system shown in blue (middle). The structure was solved by combining rotation electron diffraction (RED), HRTEM image, powder X-ray diffraction and solid state NMR.

Keywords: electron crystallography, electron diffraction, high resolution electron microscopy, crystallographic image processing, structure determination, space group determination, zeolite

\section{MS11-O5 Structural insights into the} conformation of the proline rich region of neuronal protein tau

\author{
Ondrej Cehlar ${ }^{1,2}$, Rostislav Skrabana ${ }^{1,2}$, Radovan Dvorsky $^{3}$, \\ Michal Novak ${ }^{1,2}$
}

1. Institute of Neuroimmunology, Slovak Academy of Sciences, Dubravska cesta 9, 84510 Bratislava, Slovakia

2. Axon Neuroscience SE, Dvorakovo Nabrezie 10, 81102 Bratislava, Slovakia

3. Molecular Biology II, Heinrich-Heine University, Düsseldorf, Germany

email: ondrej.cehlar@savba.sk

The Alzheimer's disease-associated protein tau is a typical representative of intrinsically disordered proteins (IDPs). Under physiological conditions, tau associates with microtubules and regulates their dynamics, whereas during the progression of neurodegeneration tau dissociates from microtubules, misfolds and deposits in brain tissue creating neurofibrillary tangles composed of paired helical filaments. The monoclonal antibody Tau5 was used as a surrogate tau protein binding partner to investigate the properties of the proline-rich region of tau molecule that contributes to its binding to microtubules. The Fab fragment of Tau 5 has been crystallized alone and in complex with 30 amino acid long tau peptide ${ }^{201} \mathrm{Gly}-\mathrm{Arg}^{230}$ [1] and both structures were solved to the $1.6 \AA$ resolution [2]. The complex structure reveals the conformation of 16 residues long tau fragment and the comparison of both structures enables to observe the changes in the antibody paratope that occurred after binding of tau peptide. The kinetics of tau peptide binding to the tau5 Fab fragment was studied by the surface plasmon resonance analysis together with the evaluation of the impact of tau phosphorylation on sites T212, T217, T220 on the binding of tau peptide to the antibody. The molecular dynamics simulation was employed to evaluate the stability of the peptide conformation and the effects of phosphorylation. Acknowledgement: This work was supported by the Slovak Research and Development Agency under the contract Nos. LPP-0038-09, APVV-0677-12 and by VEGA No. 2/0163/13.

1. Cehlar, O.; Skrabana, R.; Kovac, A.; Kovacech, B.; Novak, M., Crystallization and preliminary X-ray diffraction analysis of tau protein microtubule-binding motifs in complex with Tau5 and DC25 antibody Fab fragments. Acta Crystallogr F 2012, 68, 1181-1185.

2. Cehlar, O.; Skrabana, R.; Dvorsky R.; Novak, M., Structure of tau peptide in complex with Tau5 antibody Fab fragment. Acta Crystallogr D (submitted 04/2015) 